\title{
Eficiência Fraca, Efeito Dia-da-Semana e Efeito Feriado no Mercado Acionário Brasileiro: Uma Análise Empírica Sistemática e Robusta
}

\author{
Rosemarie Bröker Bone \\ Eduardo Pontual Ribeiro
}

\begin{abstract}
Resumo
O objetivo deste artigo é apresentar evidências sobre a hipótese de eficiência fraca no mercado acionário brasileiro. Ao contrário de outros trabalhos na área no Brasil, o método estatístico empregado busca sempre verificar se as hipóteses necessárias para a validade dos testes de hipótese são verificadas. Estudando as ações do índice da Bolsa de Valores de São Paulo no período recente, verifica-se a importância de termos auto-regressivos lineares e não lineares e dos chamados efeito dia-da-semana e efeito feriado na previsão dos retornos das ações do índice. Os resultados obtidos sugerem que na maioria das ações algum dos efeitos é verificado, com particular importância da terça-feira na previsão dos retornos.
\end{abstract}

Palavras-chaves: testes de eficiência; mercado acionário brasileiro; previsão de retornos; efeito diada-semana; testes de especificação.

\begin{abstract}
The objetctive of this paper is to present evidence of weak efficiency in the Brazilian stock market. Contrary to other studies in the Brazilian literature on the topic, the statistical procedure always test the validity of the underlying hypotheses of the test statistics employed. Based on a sample of IBOVESPA index stocks for the recent period, the relevance of linear and non-linear autoregressive terms and day-of-the-week and holiday effects to the forecast of stock returns is studied. The results suggest that in most stocks some of the mentioned effects are present, in particular a Tuesday effect.
\end{abstract}

Key words: efficiency tests; Brazilian stock market; returns forecast; day-of-the-week effects; specification tests. 


\section{INTRODUÇÃO}

A hipótese de mercados eficientes e o estudo de padrões empíricos dos retornos de ativos, em particular ações, têm atraído a atenção de vários pesquisadores, desde os anos 70. No Brasil, destacamos os estudos de Lemgruber, Becker e Chaves (1988), e mais recentemente, Costa Jr. e Lemgruber (1993) e Gava (1999).

A hipótese de eficiência de mercado no mercado acionário, na sua forma fraca, de acordo com Fama (1970, 1991), pode ser entendida como o fato de que os retornos de uma ação "são imprevisíveis em relação aos retornos anteriores, ou outras variáveis passadas” (Fama, 1991, p. 1587). Isto é, são considerados passeios aleatórios, ou fair game, na linguagem de Fama (1970), ou seja,

$$
R_{i t}=\Delta p_{i t}=\varepsilon_{i t}
$$

onde $\mathrm{R}_{\mathrm{it}}$ é o retorno de um ativo $i$ no período $t$ (medido aqui como a diferença entre os logs dos preços de fechamento, $\left.\Delta p_{i t}=I n P F_{i t}-I n P F_{i t-1}\right)$. Verifica-se a hipótese de eficiência fraca se as informações disponíveis até o período $t-1$ não auxiliam a previsão dos preços dos ativos no período $t$.

Em geral entende-se como teste da hipótese de eficiência fraca a inexistência de autocorrelação nos retornos (ou termo aleatório, em um modelo de regressão). Além disso, não podem existir padrões empíricos nos retornos dos ativos tais que gerem estratégias de ganhos consistentes ao longo do tempo. Alguns dos mais importantes padrões empíricos são os chamados efeito dia-da-semana e efeito feriado, além do efeito mês-do-ano. Por outro lado, note que a hipótese de eficiência fraca não é incompatível com lucros anormais, que seriam representados por valores extremos do termo $\varepsilon_{i}$, pouco prováveis, mas não impossíveis.

O objetivo deste artigo é apresentar evidências adicionais dos padrões empíricos das ações do índice da Bolsa de Valores de São Paulo (IBOVESPA), estudando as hipóteses de eficiência fraca e efeitos dia-da-semana e feriado para o período 1996-1999. Além de apresentar estimativas para o período recente, consideramos a principal contribuição deste artigo a proposta de teste destas hipóteses de modo sistemático e mantendo atenção quanto à especificação dos modelos.

Muitos trabalhos testam as hipóteses mencionadas sem cuidados em relação à verificação dos pressupostos necessários para os testes empregados. A não verificação das hipóteses pode fazer com que os testes empregados sejam 
ineficientes e até viesados e/ou inconsistentes. Por exemplo, Costa Jr. e Lemgruber (1993) reconhecem a possibilidade de existência de heteroscedasticidade nos retornos, mas não de autocorrelação, em um modelo para testar o efeito dia-dasemana. A presença de autocorrelação pode invalidar os resultados teóricos que baseiam os testes de hipótese, empregados pelos autores, levando a possíveis inferências errôneas.

O artigo será dividido em seis seções, além da introdução. A seguir apresentamos uma revisão dos testes de eficiência fraca no Brasil, e o objetivo na seção seguinte. Na quarta seção está o detalhamento da metodologia empírica e após a escolha da amostra. A sexta seção apresenta os resultados empíricos e a última parte as conclusões.

\section{REVISÃo dA Literatura}

As análises da eficiência de mercado tiveram como artigo canônico o de Fama (1970), que apresentou três tipos de testes de eficiência: fraca, semiforte e forte. Mais tarde, Fama (1991) alterou os nomes destes testes, mantendo os seus objetivos. O teste de eficiência forte passou a ser chamado teste de informação privada; o teste de eficiência semiforte, teste de eventos; e o teste de eficiência fraca passou a ser denominado teste de previsão. O teste de eficiência da forma fraca se refere à possibilidade da previsibilidade baseada nas informações históricas dos retornos dos ativos, que são igualmente conhecidas por todos. O teste de forma semiforte considera que as informações são públicas e absorvidas pelos agentes participantes do mercado de capitais; logo não há possibilidade de retornos anormais. O teste de forma forte divide as informações em públicas e privadas, sendo que as informações privadas se referem à possibilidade de monopólio ou acesso privilegiado (Fama, 1991; Damodaran, 1998).

Vários trabalhos estudaram a previsibilidade de retornos de ações para o mercado de ativos no Brasil, em particular as ações, conforme o Quadro 1, onde apresentamos os principais. 


\section{Quadro 1: Estudos de Efeitos Fim-de-Semana e Previsibilidade de Ativos Financeiros no Brasil}

\begin{tabular}{|c|c|c|c|c|}
\hline Autores & Amostra & Objetivos & Resultados & Método Usado \\
\hline $\begin{array}{l}\text { Lemgruber, Becker } \\
\text { e Chaves (1988) }\end{array}$ & $\begin{array}{l}\text { Preços diários de } \\
\text { fechamento do IBV } \\
\text { e IBOVESPA, ago/ } \\
83 \text { a ago/87. }\end{array}$ & $\begin{array}{l}\text { Examinar o processo } \\
\text { de geração de } \\
\text { retornos. Hipóteses: } \\
\text { (a) geração por dias } \\
\text { calendário; e (b) } \\
\text { geração por dias de } \\
\text { negócios. }\end{array}$ & $\begin{array}{l}\text { Ambas as hipóteses } \\
\text { foram rejeitadas, } \\
\text { verificando-se apenas } \\
\text { o efeito segunda- } \\
\text { feira. }\end{array}$ & $\begin{array}{l}\text { Regressão } \\
\text { múltipla com } \\
\text { variáveis dummy. }\end{array}$ \\
\hline Costa Jr. (1990) & $\begin{array}{l}\text { Preços do } \\
\text { IBOVESPA: } \\
\text { mensais, 1969- } \\
\text { 1988; diários, jan/ } \\
\text { 86-mar/89. }\end{array}$ & $\begin{array}{l}\text { Verificar se os efeitos } \\
\text { dia-da-semana e } \\
\text { mês-do-ano também } \\
\text { são encontrados no } \\
\text { mercado brasileiro } \\
\text { de ações. }\end{array}$ & $\begin{array}{l}\text { Observação do efeito } \\
\text { segunda-feira (dia- } \\
\text { da-semana) e } \\
\text { nenhum efeito mês- } \\
\text { do-ano. }\end{array}$ & $\begin{array}{l}\text { Regressão } \\
\text { múltipla com } \\
\text { variável dummy. }\end{array}$ \\
\hline $\begin{array}{l}\text { Costa Jr. e } \\
\text { O’Hanlon (1991) }\end{array}$ & $\begin{array}{l}121 \text { ações. Preços de } \\
\text { fechamento mensais } \\
\text { de } 1970 \text { a } 1990 .\end{array}$ & $\begin{array}{l}\text { Investigar a } \\
\text { existência do efeito- } \\
\text { tamanho, e se este } \\
\text { possui relação } \\
\text { significativa com } \\
\text { algum mês do ano. }\end{array}$ & $\begin{array}{l}\text { Confirmado o efeito } \\
\text { tamanho, não } \\
\text { detectado efeito mês- } \\
\text { ano. }\end{array}$ & $\begin{array}{l}\text { Retorno ajustado } \\
\text { ao mercado e ao } \\
\text { risco. }\end{array}$ \\
\hline $\begin{array}{l}\text { Costa Jr. e } \\
\text { Lemgruber (1993) }\end{array}$ & $\begin{array}{l}83 \text { ações. Preços } \\
\text { diários de abertura, } \\
\text { fechamento e média, } \\
\text { jan/86 a dez/89. }\end{array}$ & $\begin{array}{l}\text { Examinar as } \\
\text { hipóteses de } \\
\text { retornos por dia } \\
\text { calendário e dias de } \\
\text { negócios e se há } \\
\text { relação com o } \\
\text { volume de ações } \\
\text { negociadas. }\end{array}$ & $\begin{array}{l}\text { Efeito segunda-feira } \\
\text { mais acentuado para } \\
\text { ações menos } \\
\text { negociadas. Maior } \\
\text { parte do retorno das } \\
\text { mais negociadas é } \\
\text { formado fora do } \\
\text { horário de pregão. }\end{array}$ & $\begin{array}{l}\text { Regressão } \\
\text { múltipla com } \\
\text { variáveis dummy. }\end{array}$ \\
\hline $\begin{array}{l}\text { Leal e } \\
\text { Sandoval (1994) }\end{array}$ & $\begin{array}{l}\text { Retornos diários do } \\
\text { IBOVESPA, jan/82 } \\
\text { a mar/93. }\end{array}$ & $\begin{array}{l}\text { Examinar as } \\
\text { hipóteses de } \\
\text { retornos por dia-da- } \\
\text { semana, mês-do- } \\
\text { ano, virada do mês, } \\
\text { e reversão na } \\
\text { segunda-feira }\end{array}$ & $\begin{array}{l}\text { Efeito dia-da-semana } \\
\text { (segunda-feira)e } \\
\text { mês-do-ano (abril/ } \\
\text { agosto). Não há } \\
\text { efeito virada-do-mês } \\
\text { nem reversão na } \\
\text { segunda-feira. }\end{array}$ & $\begin{array}{l}\text { Análise da } \\
\text { estatística } \\
\text { descritiva. }\end{array}$ \\
\hline Gava (1999) & $\begin{array}{l}\text { Preços diários de } \\
\text { fechamento dos } \\
\text { contratos de DI } \\
\text { negociados na } \\
\text { BM\&F. Período jul/ } \\
91 \text { a out/96. }\end{array}$ & $\begin{array}{l}\text { Examinar o efeito } \\
\text { dia-da-semana por } \\
\text { meio de testes de } \\
\text { autocorrelação. }\end{array}$ & $\begin{array}{l}\text { As informações } \\
\text { passadas são } \\
\text { relevantes na } \\
\text { determinação de } \\
\text { retornos em excesso. }\end{array}$ & $\begin{array}{l}\text { Regressão } \\
\text { múltipla. }\end{array}$ \\
\hline
\end{tabular}

Fonte: baseado em Schiell (1996), com atualizações dos autores. 
Como vemos, a maioria dos estudos identificaram o efeito dia-da-semana, em particular o efeito segunda-feira. Não encontramos nenhum trabalho estudando o efeito feriado. O estudo de Becker (1989) indica a existência de retornos passados, explicando os retornos esperados para o IBOVESPA e o de Gava (1999) para os contratos de mercados futuros. Os estudos internacionais são muitos. Destacamos os citados em Fama (1991) para os Estados Unidos, além de Tan e Tat (1998) para Taiwan, Clare, Garrett e Jones (1997) para a Europa e Peña (1995) para a Espanha. Em quase todos, os efeitos dia-da-semana ou autocorrelação dos retornos, também chamados anomalias de mercados, foram verificados. Vale a pena ressaltar que a existência dessas anomalias é interpretada por muitos (não todos) pesquisadores como indicador da falência de modelos de precificação de ativos por equilíbrio, como o CAPM. Discussões detalhadas podem ser vistas em Fama (1991), Ross, Westerfield e Jaffe (1997) e Damodaran (1998), por exemplo.

\section{Objetivo}

O objetivo deste artigo é verificar as evidências quanto à validade de modelos de preços de equilíbrios de ativos financeiros em relação a sua eficiência fraca, para o mercado brasileiro, em especial para as ações participantes no índice da Bolsa de Valores de São Paulo (IBOVESPA), usando-se o preço de fechamento diário durante o período de 1996 a 1999 (4 de janeiro - 29 de outubro).

O objetivo secundário é apresentar um método de estudo empírico em linha com a moderna teoria econométrica, desenvolvida nos últimos 20 anos, com o uso difundido de testes de especificação do modelo empírico ainda muito pouco explorado na literatura da Administração.

Acredita-se que esse exercício irá contribuir para o enriquecimento da literatura sobre a eficiência de mercado no Brasil e apresentará evidências do efeito diada-semana e do retorno defasado na precificação dos ativos que compõem o IBOVESPA.

\section{Metodologia Empírica}

\section{Detalhamento dos Testes de Eficiência Fraca}

A análise centra-se nos testes sobre a eficiência fraca. Esta análise não será baseada em modelo teórico para verificação empírica, mas em modelo exploratório 
do comportamento dos dados. Em geral, a hipótese de eficiência fraca é equivalente à verificação de que o passado dos retornos não pode ajudar a prever os retornos futuros, ou seja, $H_{0}$ : $E\left(R_{t} \mid I_{t-1}\right)=E\left(R_{t}\right)$, onde $I_{t-1}$ representa o conjunto de informação disponível para os investidores no tempo $t-1$. Em outras palavras, a hipótese indica que os retornos esperados condicionais à informação passada não são diferentes dos retornos esperados não condicionais, ou seja, que desprezam o comportamento anterior dos retornos.

Um modo de testar esta hipótese é testar, em um modelo autoregressivo como

$$
R_{t=i t}=\alpha+\beta R_{i t-1}+\varepsilon_{t},
$$

se $H_{0}: \beta=0$. Vale a pena ressaltar que este teste é diferente de um teste da hipótese em que os retornos possuem valor esperado zero, ou seja, $E\left[R_{i t} \mid R_{i t-1}\right]=0$, como, por exemplo, indica Gava (1999). Na verdade, como sob hipóteses usuais (em particular, $|\beta|<1$, veja por exemplo, Maddala, 1996),

$$
E\left[R_{i t} \mid R_{i t-1}\right]=\alpha+\beta R_{i t-1} \quad \text { e } \quad E\left[R_{i t}\right]=\alpha /(1-\beta) .
$$

Se a hipótese nula é verificada, $E\left[R_{i t} \mid R_{i t-1}\right]=E\left[R_{i t}\right]=\alpha$. (implicitamente estamos assumindo que a veracidade da hipótese se deve à independência temporal de $R_{i t}$ ). O fato de a média ser diferente de zero não invalida o modelo CAPM, por exemplo, a não ser que o mercado e o ativo sem risco também tenham retornos esperados zero. O importante é que os retornos sejam imprevisíveis a cada dia, a não ser pelo uso de sua média histórica (Fama, 1991).

Ao mesmo tempo, uma importante preocupação nos modelos CAPM é a distribuição dos retornos. Como nos mostra Varian (1992), a validade do modelo CAPM como gerador dos retornos de ativos em um mercado passa pela hipótese de que os retornos seguem uma distribuição normal (gaussiana). Em outras palavras, omitindo um índice i para cada ativo para poupar notação,

$$
R_{t} \sim N\left(\mu, \sigma^{2}\right)
$$

onde $\mu$ é a média dos retornos e $\sigma^{2}$ a variância do ativo em estudo.

Para testar a hipótese de normalidade, podemos empregar alguns testes de normalidade como o de Jarque e Bera (apud Hill, Grifiths e Judge, 1999); todavia este e outros testes partem do pressuposto de que a amostra empregada é iid (independente e identicamente distribuídos), sendo que violações desta hipótese podem levar a graves erros de decisão (tipo I e tipo II) no teste de hipótese. Assim, é importante garantir que os retornos são iid, ou condicionalmente iid. Para isso é necessário especificar corretamente um modelo amplo de previsão dos retornos esperados. 
Pelas propriedades estatísticas, podemos utilizar a seguinte representação alternativa de [1]:

$$
R_{t}=\alpha+\varepsilon_{t}
$$

onde $\varepsilon_{t} \sim N\left(0, \sigma^{2}\right), \alpha$ representa a média de $R_{t}$ e $\varepsilon_{t}$ um termo aleatório. Pode ser o caso de que esta média dependa do tempo, ou que $\alpha$ seja variante no tempo. $\mathrm{Na}$ literatura internacional os chamados efeitos-calendário são aceitos como fatos estilizados da distribuição de retornos de uma ação, na forma de efeito janeiro, efeito feriado ou efeito dia-de-semana (Ross, Westerfield e Jaffe [1997], Damodaran [1999] e outros) como mencionado acima. Se em algum dia-dasemana os retornos forem sistematicamente maiores (ou menores) que em outros dias, o termo aleatório passa a ter valores extremos incompatíveis com a distribuição normal, levando à perda de eficiência, e até viés, nos testes de hipótese de normalidade baseados em [1']. O mesmo ocorre com testes não-paramétricos, como Kolmogorov-Smirnov e Wilcoxon.

Em nosso caso, como estamos estudando o comportamento recente (19961999) dos ativos, não temos graus de liberdade suficientes para estimar o efeito mês-do-ano com confiança. Nosso interesse enfoca-se no efeito dia-da-semana e feriado, construindo assim a equação de retornos

$$
R_{t}=f(\text { dia-da-semana, feriado })+\varepsilon_{t}
$$

com $\varepsilon_{t} \sim i i d N\left(0, \sigma^{2}\right)$. Ou seja, $R_{t}-f($ dia-da-semana, feriado $)=\varepsilon_{t} \sim N\left(0, \sigma^{2}\right)$. Assumindo um efeito linear sobre o log-retorno, temos,

$$
R_{t}=\alpha+\beta_{1} \operatorname{Seg}+\beta_{2} \text { Ter }+\beta_{3} \text { Quin }+\beta_{4} \operatorname{Sex}+\beta_{5} \text { vesp }+\beta_{6} \text { após }+\varepsilon_{t}
$$

com $\varepsilon_{t} \sim i i d N\left(0, \sigma^{2}\right)$. Seg, Ter, Quin e Sex são variáveis indicativas (binárias ou dummy), que tomam valor 1 nos respectivos dias da semana (e 0 em outros dias), e medem as diferenças de retornos médios entre os respectivos dias e a quartafeira $(\alpha)$. vesp e após são dummies que representam dias de véspera e após feriados e medem diferenças de retornos médios em relação a outros dias úteis.

Para completar o modelo empírico, este tem de levar em conta a possibilidade de violação da hipótese de eficiência fraca pela dependência temporal dos retornos, ou seja, $E\left(R_{t} \mid I_{t-1}\right) \neq E\left(R_{t}\right)$, onde $I_{t-1}$ representa o conjunto de informações disponíveis para os investidores no tempo t-1. Em outras palavras, as distribuições condicional e não condicional dos retornos em relação ao passado são diferentes. Esta característica será incluída no nosso modelo, que compreende o retorno defasado de um período. A rationale para a inclusão do retorno esperado na 
equação de previsão de retornos é que o preço do ativo defasado é um resumo satisfatório da informação disponível aos investidores.

É curioso notar que testes para efeitos sazonais (dia-da semana etc.) no Brasil, como por exemplo Leal e Sandoval (1994), identificam que os erros são autocorrelacionados, mas não reconhecem que a autocorrelação no resíduo pode ser sintoma de autocorrelação na variável dependente. Seguindo a moderna teoria econométrica, entendemos ser melhor (em termos de eficiência) incluir uma variável dependente defasada do que modelar um erro autocorrelacionado per se. Uma argumentação detalhada pode ser encontrada em Hendry (1994).

O modelo empírico utilizado para a parte empírica do trabalho é dado pela equação abaixo:

$$
\begin{aligned}
R_{t}= & \alpha+\beta_{1} \operatorname{Seg}+\beta_{2} \text { Ter }+\beta_{3} \text { Quin }+\beta_{4} \text { Sex }+\beta_{5} \text { vesp }+ \\
& \beta_{6} \text { após }+\beta_{7} R_{t-1}+\varepsilon_{t},
\end{aligned}
$$

com a hipótese que $\varepsilon_{t} \sim i i d N\left(0, \sigma^{2}\right)$. Uma versão da hipótese de eficiência fraca é verificada se todos os coeficientes betas forem estatisticamente iguais a zero, além de os erros terem uma distribuição normal. Uma segunda versão da hipótese de eficiência fraca requer apenas que os coeficientes beta sejam iguais a zero, ou seja, os retornos sendo imprevisíveis em relação à informação passada ou características do dia.

Recentemente, vários artigos têm identificado que os retornos das ações brasileiras possuem variância condicional variável, ou seja, o termo aleatório do modelo [3] acima segue um modelo ARCH (autoregressive conditional heteroscedasticity), como, por exemplo, os de Bertucci (1999) ou Galvão, Portugal e Ribeiro (2000).

Devemos testar também a possibilidade de que o termo aleatório tenha padrão ARCH; todavia note que uma comparação das equações de testes para as hipóteses de:

- heteroscedasticidade de $R_{t}$ em um modelo autorregressivo - teste de White no formato Lagrange Multiplier ou LM (o quadrado do resíduo em função de $R_{t-1}$ e seu quadrado),

. não linearidade - teste LM para não linearidade, também chamado RESET (o resíduo em função do quadrado de $R_{t-1}$ ), e

- erros da forma ARCH(p) - teste LM para erros ARCH (o quadrado do resíduo em função do quadrado do resíduo defasado até a ordem $p$ ), 
nos leva a concluir que são muito similares em um modelo como o [3], podendo, na prática, levar à rejeição da hipótese nula em todos os casos, quando apenas uma delas é efetivamente falsa. Dizemos que os testes são observacionalmente similares. Note que a não linearidade pode gerar heteroscedasticidade em um modelo de regressão linear (Maddala, 1996) e que a única variável explicativa é o retorno defasado, um componente autoregressivo.

Dessa forma, nosso método empírico será testar heteroscedasticidade por meio do teste de White na equação [3]; se rejeitada, incluir na equação o termo $\left(R_{t-1}\right)^{2}$, fazendo que o modelo final seja

$$
\begin{aligned}
R_{t}= & \alpha+\beta_{1} \text { Seg }+\beta_{2} \text { Ter }+\beta_{3} \text { Quin }+\beta_{4} \text { Sex }+\beta_{5} \text { vesp }+ \\
& \beta_{6} \text { após }+\beta_{7} R_{t-1}+\beta_{8}\left(R_{t-1}\right)^{2}+\varepsilon_{t},
\end{aligned}
$$

com a hipótese que $\varepsilon_{t} \sim \operatorname{iid} N\left(0, \sigma^{2}\right)$. Testes ARCH também serão levados a cabo, mas se tais erros forem verificados em [3], o ajuste se dará via a equação [4]. Para garantir a validade das estatísticas $t$ e dos testes F (testes de Wald, se considerarmos a distribuição dos resíduos não-normal), testes de heteroscedasticidade e autocorrelação serão calculados, mais uma vez, para [4]; se as hipóteses forem rejeitadas, empregaremos matrizes de variância/covariância dos parâmetros robustas de White e Newey-West (ver, por exemplo, Greene [1996]).

O uso das matrizes de variância-covariância robustas busca garantir que os testes de hipótese sobre os coeficientes tenham propriedades estatísticas desejadas. Note também que no caso de a hipótese de normalidade ser rejeitada, os testes de hipótese passam a ter justificativa assintótica para grandes amostras. Como as amostras incluem mais de 700 observações, espera-se que as propriedades assintóticas estejam válidas, ou seja, os desvios da distribuição das estatísticas em relação às de grandes amostras, que diminuem com o tamanho da amostra e são zero, quando a amostra é infinita, sejam próximos de zero. No caso da distribuição dos erros não ser normal, os testes de hipótese aqui utilizados, passam a ter uma interpretação de testes baseados em estimadores de quase-máxima verossimilhança, que possuem boas propriedades estatísticas em grandes amostras (Greene, 1996).

Last but not least, note que se em um modelo como [3], com uma variável endógena defasada, tivermos autocorrelação dos resíduos, os coeficientes estimados por MQO (ou até MQP, mínimos quadrados ponderados) serão viesados e inconsistentes em grandes amostras. Dois pontos importantes cumpre notar. $\mathrm{O}$ teste de autocorrelação em [3] não pode ser o teste Durbin-Watson, e a autocorrelação pode ser devido à omissão de $R_{t-2}$, por exemplo. Assim, testamos 
autocorrelação em [3], utilizando o teste de autocorrelação de Breusch-Godfrey (Maddala, 1996); se rejeitado, incluímos $R_{t-2}$ em [3], ou [4].

Nosso interesse em especificar um modelo de regressão para os retornos se deve à busca de um modelo que garanta que os resíduos sejam efetivamente iid. Outros artigos (Leal e Sandoval [1994], por exemplo) utilizam testes nãoparamétricos como os de Mann-Whitney e Kruskall-Wallis, pois "não exigem que os dados tenham um forma de distribuição pré-determinada” (Leal e Sandoval, 1994, p. 215). Mas note que os testes, apesar de não exigirem normalidade dos resíduos, exigem que estes sejam iid, o que não é verdade na presença de heteroscedasticidade e/ou autocorrelação. Alguns autores preocupam-se com autocorrelação, mas não com heteroscedasticidade. De qualquer forma, as hipóteses necessárias para validar os testes empregados não são verificadas, o que pode gerar resultados errôneos na suas aplicações. Por outro lado, a justificativa de modelar heteroscedasticidade temporal e/ou a autocorrelação na equação, ao contrário de métodos como mínimos quadrados ponderados (MQP), deve-se à possível perda de eficiência na estimação de MQP na prática.

\section{Detalhamento do Teste de Wald}

O teste de Wald para a hipótese de um coeficiente apenas igual a zero, em regressão simples ou múltipla, estimada por MQO (mínimos quadrados ordinários) ou MQG (mínimos quadrados generalizados), equivale ao quadrado das estatísticas $t$ usualmente apresentadas por pacotes estatísticos. No caso de erros iid, o teste de Wald para restrições simultâneas em mais de um coeficiente pode ser calculado como teste $F$. Embora a distribuição em grandes amostras da estatística de Wald sob a hipótese nula se aproxime da distribuição qui-quadrado, é possível demonstrar que uma variável aleatória $F$, multiplicada pelos graus de liberdade do numerador de sua expressão, segue uma distribuição qui-quadrada com estes mesmos graus de liberdade. Assim há equivalência entre os testes Wald e $F$. Na prática, os testes de Wald empregam a tabela $F$, com os ajustes apropriados das estatísticas, com justificativas de ajustes para pequenas amostras, como em Hendry (1994).

No caso de erros não serem da forma iid, o teste de Wald possui uma forma elaborada, que pode ser encontrada em vários livros de econometria avançada, como por exemplo, o de Greene (1996). Grosso modo, o teste é baseado no produto quadrado do vetor de coeficientes (estimados de forma consistente) com uma matriz de restrições das hipóteses em estudo e o inverso da matriz de variânciacovariância dos coeficientes. Como o teste requer apenas estimadores consistentes dos coeficientes, no caso de erros heterocedásticos e/ou autocorrelacionados, os mesmos podem ser estimados via MQO. Neste caso, para o cálculo do teste, um ajuste na matriz de variância dos coeficientes é ne- 
cessário. Estes ajustes são não-paramétricos, no sentido de não dependerem de forma específica de heteroscedasticidade ou autocorrelação (ou até a distribuição dos erros) e assim robustos a erros na especificação da forma dos erros para MQG. Empregamos a matriz de Newey-West para erros autocorrelacionados e heterocedásticos e o caso especial - a matriz de White - para erros apenas heterocedásticos. Em suma, chamamos atenção ao fato de que a metodologia também é robusta, no sentido de que diferentes especificações na forma dos erros (forma de heteroscedasticidade e ordem de autocorrelação) são acomodadas pelos procedimentos aqui empregados.

\section{Detalhamento do Teste de White para Heteroscedasticidade}

O teste de White, baseado no princípio do multiplicador de Lagrange, ou do score (Maddala, 1996), tem o propósito de verificar a presença de heteroscedasticidade. Para isso a sugestão é fazer uma regressão dita auxiliar, cuja variável dependente seja a estimativa dos erros ao quadrado ( $\left.\hat{\varepsilon}^{2}\right)$ e as variáveis explicativas (independentes) sejam seus quadrados e seus produtos cruzados. Sob certas hipóteses usuais, pode-se demonstrar que $n R^{2}$ (onde $R^{2}$ é o coeficiente de determinação total ou múltiplo e $n$ o número de observações) desta regressão auxiliar segue uma distribuição $\chi^{2} \operatorname{com} p$ graus de liberdade, onde $p$ é o número de coeficientes estimados na regressão auxiliar.

\section{Detalhamento do Teste LM (Breusch e Godfrey) para Autocorrelação}

Este teste acomoda casos mais gerais para a verificação de autocorrelação, como, por exemplo, modelos com variável endógena defasada (ao contrário dos testes Durbin-Watson e $Q$ de Lijung e Box) e autocorrelação de ordem superior a um, ou até de erros do tipo média móvel. Este teste também deriva do princípio do multiplicador de Lagrange (Madalla, 1996).

Testa-se a hipótese nula de inexistência de correlação dos erros de ordem $p$ por meio da regressão dos resíduos de [3]; por exemplo, em função destes resíduos defasados 1 até $p$ períodos e todas as variáveis explicativas do modelo. $n R^{2}$ desta regressão segue, como acima, uma distribuição $\chi^{2}$ com $p$ graus de liberdade, onde $p$ é a ordem de autocorrelação suposta.

\section{Escolha da Amostra}

A escolha da amostra levou em conta as ações pertencentes ao IBOVESPA 
referentes ao período de janeiro 1996 a dezembro de 1998. A escolha das ações se deve ao uso daquelas ações mais importantes no mercado acionário brasileiro em termos de liquidez. Ações menos liquidas não são transacionadas com freqüência, o que dificulta a análise do comportamento diário. Escolhemos certa perda de generalidade dos resultados ao não escolher todas as ações transacionadas na BOVESPA, em troca de evitar dificuldades com preços inexistentes ao longo do tempo, bem como o curto período de vigência em relação ao período amostral. Por isso, das ações pertencentes ao IBOVESPA retiramos as seguintes ações: ELETROPAULO PN (que estreou no pregão em 31/3/98), GERASUL ON (1/6/ 98), TELESP Cel. PNB (18/5/98), Geradora Tietê PN (26/7/99), Geradora Paranapanema PN (26/7/99), Transmissão PN (26/7/99) e TELESP PN (21/9/98). A escolha do período se deve ao interesse do comportamento das ações após o período do Real, com clara estabilidade de preços, sem também incluir as mudanças de regime cambial de janeiro de 1999, que muito certamente geraram quebras estruturais no processo gerador dos retornos.

Seguimos Mayers e Rumsey (apud Vieira e Procianoy, 1998) e calculamos o retorno no período $t$ como a diferença dos log dos preços de fechamentos no dia $t$ e $t-1$, ou o último período anterior em que a ação foi transacionada, seja devido à fins-de-semana, feriados, ou à não transação da ação. Como escolhemos apenas as ações do IBOVESPA, que, por construção são as mais líquidas do mercado, o número de vezes em que o último ocorre é pequeno.

\section{Resultados Empíricos}

Os resultados são apresentados nas Tabelas 1 e 2. Decidimos apresentar tabelas resumidas, pois estamos trabalhando com 38 ações. Os resultados completos estão disponíveis com os autores. Em todas as ações os testes de normalidade dos resíduos de Jarque-Bera rejeitaram tal hipótese. Assim, todos os testes apresentados passam a ter justificativa apenas assintótica, como mencionado acima. Observe-se que os testes empregados são relativamente robustos nesta não-normalidade dos resíduos, como mostra Greene (1996) e, principalmente, White (1980). Na Tabela 1 apresentamos os sinais dos efeitos dia-da-semana e feriado e termos autoregressivos para aqueles coeficientes significativos até $10 \%$. O modelo citado refere-se aos últimos estimados, ou seja, [3] se não há autocorrelação ou heteroscedasticidade nos modelos, [4] se há heteroscedasticidade, e [3] e [4] com matrizes de variânciacovariância dos parâmetros ajustados para autocorrelação e autocorrelação e heteroscedasticidade, respectivamente, se houve apenas autocorrelação ou se em [4] os testes indicaram a presença de autocorrelação e/ou heteroscedasticidade após a correção (inclusão de $R_{i t-1}{ }^{2}$ ). 


\section{Tabela 1: Sinais e Significância dos Efeitos Dia-da-Semana, Feriado e Termos Autorregressivos}

\begin{tabular}{|c|c|c|c|c|c|c|c|c|c|c|c|}
\hline Ações\Coef. & Constante & \begin{tabular}{|l|} 
Segunda \\
\end{tabular} & Terça & Quinta & Sexta & \begin{tabular}{|l|l|} 
Véspera \\
\end{tabular} & Após & $\operatorname{Ret}(\mathrm{t}-1)$ & $\operatorname{Ret}(\mathrm{t}-1)^{2}(\mathrm{a})$ & $\begin{array}{c}\text { Testes } \\
\text { Espec.(b) }\end{array}$ & $\begin{array}{c}\text { Correção } \\
\text { (c) }\end{array}$ \\
\hline Aracruz Pnb & & & & & & & & - & - & AC/HET & \\
\hline Banespa Pn & & & & - & & & & + & + & HET & HET \\
\hline Bradesco Pn & - & & + & & + & & & + & + & HET & HET \\
\hline Brahma Pn & & & + & & & & & + & & HET & HET \\
\hline Brasil On & & - & & & & & & & & HET/AC & HET \\
\hline Brasil Pn & & & & & & & & + & & $\mathrm{AC}$ & HET/AC \\
\hline Brasmotor & & & & & + & & & + & & HET & HET \\
\hline Celesc Pnb & & & & & & + & & + & & HET & HET \\
\hline Cemig On & & & & - & & & & + & & HET & HET \\
\hline Cemig Pn & & & & & & & & + & + & HET & HET/AC \\
\hline Cesp Pn & & & & & & & & + & & HET & HET \\
\hline Copel Pnb & & & + & & & & & + & & HET & HET \\
\hline Copene Pna & & & & & & & + & 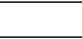 & & HET & HET \\
\hline Copesul On & & & + & & & & + & - & - & HET/AC & HET/AC \\
\hline Duratex Pn & & & & - & & & + & + & & HET & HET \\
\hline Eletrobras On & - & & + & & + & + & & + & & HET & HET \\
\hline Eletrobras Pnb & & & + & & + & & & & & HET & HET \\
\hline Inepar Pn & - & & + & & + & & & & & HET & HET \\
\hline Ipiranga Pet Pn & & & & - & & & + & & & HET & HET \\
\hline Itaubco $\mathrm{Pn}$ & & & + & & + & + & & + & & HET/AC & HET \\
\hline Itausa & & & + & & & & & & + & HET & HET \\
\hline Klabin Pn & & & & & & & & & & HET & HET \\
\hline Light On & & & & - & & & & + & & HET & HET \\
\hline Paulo Fluz On & & & + & & & & & + & & HET/AC & HET \\
\hline Petrobras Pn & - & & + & & + & & & & & HET & HET \\
\hline Petrobras-Br Pn & & & + & & & + & & + & & HET/AC & HET \\
\hline Sid.Nacional On & & & & & + & + & & & + & HET & HET \\
\hline Sid.Tubarao Pn & & & & & & & + & & & HET & HET \\
\hline Souza Cruz On & & & & & & & & & & HET/AC & HET/AC \\
\hline Telepar Pn & & & & & & & & + & & HET & HET \\
\hline Telerj Pn & & & & & & & & & & HET & HET \\
\hline Telebras 31 Ron & & & + & & & & & & & HET & HET \\
\hline Telebras $41 \mathrm{Rpn}$ & & & + & & & & & & & HET & HET \\
\hline Telesp On & & & + & & & & & + & + & HET & HET/AC \\
\hline Usiminas Pna & - & + & + & & + & & & & & HET & HET \\
\hline Votorantim Pn & & - & & - & & & & & & HET/AC & HET/AC \\
\hline Vale Rio Doce Pna & & & + & - & & & & & & HET & HET \\
\hline White Martins On & & & & - & & & + & & + & HET & HET \\
\hline
\end{tabular}

Fonte: cálculos dos autores.

Notas: se $\mathrm{p}<0,10$ : apresenta-se o sinal do coeficiente; se $\mathrm{p}>0,10$ : deixa-se em branco, onde $\mathrm{p}$ é o valor-p do teste. (a) incluído após teste de heteroscedasticidade de White na equação [3]; (b) indica a presença de heteroscedasticidade e/ou autocorrelação na equação [3]; (c) correção na matriz de variância-covariância da equação [4] após testes de especificação. HET: indica uso da correção de White na matriz de variância-covariância dos coeficientes para heteroscedasticidade; HET/AC: indica uso da correção de Newey-West para heteroscedasticidade e autocorrelação. 
Vemos que para a quase totalidade das ações foi identificada heteroscedasticidade nos resíduos e, em uma minoria, autocorrelação dos resíduos de segunda ordem. Toda a ação que teve rejeitado o teste de especificação de homoscedasticidade na equação [3], teve reestimado seu modelo empírico por meio da equação [4]. Em poucos casos o termo autoregressivo quadrático foi significativo; na maioria das vezes, não acomodou totalmente a heterogeneidade das variâncias. Todavia decidiu-se mantê-lo, pois o grau de heterogeneidade diminuiu perceptivelmente; em poucas vezes, o teste conjunto de significância indicava que os termos autoregressivos deveriam ser retirados.

O termo autorregressivo foi significativo em aproximadamente metade dos ativos estudados, sendo as estimativas do coeficiente entre 0,10 e 0,20. Isto indica pouca persistência de mudanças dos preços, visto que, passados dois períodos, um crescimento do retorno do ativo em $1 \%$ se reflete em apenas 0,01 a $0,04 \%$ sobre o retorno dois períodos à frente. Os $R^{2}$ são baixos, indicando uma preponderância do termo aleatório sobre o comportamento dos retornos das ações estudadas no período em apreciação.

Observando os resultados de testes conjuntos dos termos autoregressivos na Tabela 2, os resultados da importância dos termos autoregressivos são confirmados. Concluímos então que a evidência em relação à hipótese de eficiência fraca (autocorrelação dos retornos) é mista, sendo rejeitada em metade dos casos; mesmo assim, a parte explicada dos retornos é relativamente baixa, dado o coeficiente de determinação observado.

Quanto ao efeito dia-da-semana, na Tabela 1 verificamos que terça-feira é o dia de retornos mais diferenciados em relação à quarta-feira. Não parece que os retornos de quarta (na constante) e segunda são, em média, diferentes de zero e diferentes entre si. O efeito sexta-feira foi verificado como positivo, para poucas ações também (apenas 9). Estes resultados são diferentes dos obtidos anteriormente na literatura e listados no Quadro 1.

Por outro lado, a quinta-feira aparece como um dia de baixas em relação à quarta-feira (e segunda), embora em poucas ações. Poder-se-ia associar isso ao popularmente conhecido dia do boato em Brasília, mas o baixo número de ações com este efeito indica pouca validade desta conjectura. O maior destaque entre os dias da semana é visto na terça-feira, onde metade das ações teve retornos maiores que a segunda-feira, na média. Dentro de uma interpretação política dos resultados, poder-se-ia associar este retorno positivo diferenciado na terçafeira como um efeito Brasília, pois terça-feira é o dia associado com efetiva participação parlamentar no Congresso Nacional. 


\section{Tabela 2: Testes Conjuntos (Joint Test) dos Coeficientes}

\begin{tabular}{|c|c|c|c|c|}
\hline Ações\Coef. & $\begin{array}{l}\text { Efeito dia- } \\
\text { da-semana } \\
\text { (a) }\end{array}$ & $\begin{array}{l}\text { Efeito } \\
\text { Feriado } \\
\text { (b) }\end{array}$ & $\begin{array}{c}\text { Variáveis } \\
\text { Autoregressivas } \\
\text { (c) }\end{array}$ & $\begin{array}{c}\text { Todos os coef. } \\
\text { Exceto a } \\
\text { constante }\end{array}$ \\
\hline Aracruz Pnb & & $*$ & $* * *$ & $* * *$ \\
\hline Banespa Pn & & & $* *$ & $* * *$ \\
\hline Bradesco Pn & $* * *$ & & $* * *$ & $* * *$ \\
\hline Brahma Pn & $* * *$ & & $* * *$ & $* * *$ \\
\hline Brasil On & & & & $*$ \\
\hline Brasil Pn & & & $*$ & \\
\hline Brasmotor & & & $*$ & $* * *$ \\
\hline Celesc Pnb & $*$ & $*$ & $* *$ & $* * *$ \\
\hline Cemig On & & & $*$ & $* *$ \\
\hline Cemig Pn & & & $* * *$ & $* * *$ \\
\hline Cesp Pn & & & $* *$ & $* * *$ \\
\hline Copel Pnb & $*$ & & $* * *$ & $* * *$ \\
\hline Copene Pna & & $* * *$ & & $* *$ \\
\hline Copesul On & $* * *$ & $* * *$ & $* * *$ & $* * *$ \\
\hline Duratex Pn & $* *$ & $* * *$ & $* * *$ & $* * *$ \\
\hline Eletrobras On & $* *$ & & & $* * *$ \\
\hline Eletrobras Pnb & $*$ & & & $* * *$ \\
\hline Inepar Pn & $* *$ & & & $* *$ \\
\hline Ipiranga Pet Pn & & $*$ & & \\
\hline Itaubco Pn & $* * *$ & $*$ & $* * *$ & $* * *$ \\
\hline Itausa & $*$ & & & $* *$ \\
\hline Klabin Pn & & & & $*$ \\
\hline Light On & & & & $* *$ \\
\hline Paulo Fluz On & $*$ & & $* * *$ & $* * *$ \\
\hline Petrobras Pn & $* * *$ & & $*$ & $* * *$ \\
\hline Petrobras-Br Pn & $* * *$ & $*$ & $*$ & $* * *$ \\
\hline Sid.Nacional On & $* *$ & & $* * *$ & $* * *$ \\
\hline Sid.Tubarao Pn & $* *$ & $* *$ & & $* *$ \\
\hline \multicolumn{5}{|l|}{ Souza Cruz On } \\
\hline Telepar Pn & & & $* * *$ & $* * *$ \\
\hline Telerj Pn & $*$ & & & $* *$ \\
\hline Telebras 31 Ron & $* *$ & & & $* * *$ \\
\hline Telebras 41 Rpn & $* *$ & & & $* *$ \\
\hline Telesp On & $*$ & & $* * *$ & $* * *$ \\
\hline Usiminas Pna & $* * *$ & & & $* * *$ \\
\hline Votorantim Pn & $* *$ & & & $* *$ \\
\hline Vale Rio Doce Pna & $* *$ & & & $* *$ \\
\hline White Martins On & $* * *$ & & $* * *$ & $* * *$ \\
\hline
\end{tabular}

Notas: se $p<0,01$ : **; se $0,01<p<0,05$ : **; se 0,05<p<0,10: *, onde $p$ é o valor $p$ do teste bicaudal. Os testes baseados na equação [4] ou [3] com matriz de variância-covariância indicada na Tabela 1.

(a) $H_{0}: \beta_{1}=\beta_{2}=\beta_{3}=\beta_{4}=0$ na equação [4];

(b) $\mathrm{H}_{0}: \beta_{5}=\beta_{6}=0$ na equação [4];

(c) $H_{0}: \beta_{7}=\beta_{8}=0$ na equação [4] ou $\beta_{7}=0$ na equação [3] se não há HET/AC;

(d) $\mathrm{H}_{0}: \beta_{1}=\ldots=\beta_{8}=0$. 
Observando os resultados dos testes de hipótese conjunta de que todos os dias da semana têm coeficientes iguais a zero na Tabela 2, a importância do dia da semana para ajudar a prever os retornos (incluindo a constante, que se refere à quarta-feira) é confirmada para mais da metade das ações (24 em 38 ações), como em outros trabalhos. Mas note que existe um diferencial dos resultados obtidos em comparação aos da literatura nacional e internacional, que indica a existência, basicamente, de um efeito segunda-feira. Nossos resultados indicam um diferencial de retornos, em média, de terça-feira.

Passando, por último, para a análise dos resultados referentes ao efeito feriado, identificamos poucas ações em que os retornos nas vésperas e após feriados são diferenciados em relação aos outros tipos de dias de pregão. Nas poucas ações em que tal efeito foi verificado, estes são positivos. Em nenhuma ação ambos os pregões antes e depois de feriados são individualmente diferenciados. E para 29 ações, baseados nos resultados apresentados na Tabela 2, não podemos rejeitar a hipótese de que nem a véspera nem o dia após um feriado possuem retornos, em média, diferenciados dos outros dias de operação da BOVESPA.

\section{Conclusões}

O objetivo deste artigo foi apresentar evidências adicionais sobre as diferentes formas da hipótese de eficiência fraca no mercado brasileiro de ações, a saber, a previsibilidade de retornos baseados nos retornos passados, e patamares de retornos diferenciados por características de dias de pregão (dia da semana e próximos a feriados). Nossa proposta de estudo busca acompanhar um método de especificação de um modelo empírico, cujas hipóteses sobre o termo aleatório sejam estudadas e não apenas tomadas como verdadeiras; em particular, heteroscedasticidade e/ou autocorrelação, e normalidade dos resíduos, além da forma funcional, com o uso de instrumental econométrico moderno. Tal abordagem é quase inexistente na literatura de finanças nacionais, principalmente em revistas de administração.

Verificamos que para aproximadamente metade das ações estudadas o passado dos retornos auxilia na previsão dos retornos das ações do IBOVESPA, embora a parte da variabilidade dos retornos, explicada pelos termos autoregressivos, seja bem pequena. Verificamos, também, que existe efeito dia-da-semana em aproximadamente metade das ações estudadas, sendo o dia mais diferenciado, em termos de retornos médios, a terça-feira, ao que apelidamos efeito Brasília. Por outro lado, vésperas e dias seguintes a feriados possuem retornos médios diferenciados para uma pequena minoria de ativos apenas. 
Em suma, todas as ações do índice IBOVESPA estudadas, exceto Brasil Pn, Ipiranga Pet Pn e Souza Cruz On, apresentam algum tipo de violação da hipótese de eficiência fraca no mercado de ações, seja ela devida ao efeito dia-da-semana, efeito feriado ou termos autoregressivos, com base nos resultados obtidos neste trabalho.

\section{REFERÊNCIAS BiblográficAs}

BECKER, J. L.

Teste de eficiência fraca do mercado de capitais. Porto Alegre, PPGA/UFRGS. mimeo, s.d. 1989.

BERNDT, E.

The practice of econometrics: classic and contemporary. New York: Addison-Wesley, 1991.

BERTUCCI, L. A.

Avaliação de modelos de volatilidade condicionada na precificação de opções de compra no mercado da BOVESPA. In: ENCONTRO ANUAL DA ANPAD, XXIII, 1999, Foz do Iguaçu. Anais... Foz do Iguaçu: ANPAD, 1999. 1 CD-ROM.

BRITO, N.

Eficiência informacional fraca de mercado de capitais sob condições de inflação. Revista Brasileira de Mercado de Capitais, v. 4, n. 10, p. 30-44, 1978.

CLARE;

GARETT;

JONES.

Testing for seasonal patterns in conditional retourn volatility. Applied Financial Economics, v. 7, n. 3, p. 517-523, 1997.

COSTA JR., N. C.;

LEMGRUBER, E. F.

$O$ efeito fim de semana durante períodos de abertura e de fechamento das bolsas de valores. In: ENCONTRO ANUAL DA ANPAD, XVII, 1993, Salvador. Anais... Salvador: ANPAD, 1993. p. 103-110.

DAMODARAN, A.

Avaliação de investimentos.

Rio de Janeiro: Qualitymark, 1999.

DELGADO, S.

Distribuição do retorno das ações no Brasil. Revista de Econometria, 1996.

FAMA, E.

Efficient capital markets: a review of theory and empirical work. Journal of Finance, v. 25, n. 1, p. 383-470, 1970.

Efficient capital markets II. Journal of Finance, v. 46, n. 5, p. 1575-1617, 1991. 
GALVÃO, A. B.;

PORTUGAL, M.;

RIBEIRO, E. P.

Causalidade na volatilidade entre os mercados à vista e futuros do IBOVESPA.

Revista

Brasileira de Econometria, 2000.

GAVA, A.

Mercado futuro brasileiro: distribuição estatística e eficiência das previsões do contrato futuro de DI. Análise, v. 10, n. 1, p. 1935, 1999.

GREENE, W.

Econometric theory. 3. ed. New York: McGraw-Hill, 1996.

HENDRY, D.

Dynamic econometrics. Oxford: University Press, 1994.

HILL, C.;

GRIFFITHS, W.;

JUDGE, G.

Econometria. São Paulo: Saraiva, 1998.

LEAL, R. P.;

SANDOVAL, E. B.

Anomalias nos mercados de ações de países em desenvolvimento. In: ENCONTRO ANUAL DA ANPAD, XVIII, 1994, Curitiba. Anais... Curitiba: ANPAD, 1994. p. 213-230.
MADDALA, G. S.

Introducción a la econometria.

2. ed. Mexico: McGraw-Hill, 1996.

MATOS, O.

Econometria básica. São Paulo: Atlas, 1997.

PEÑA, J.

Daily seasonalities and stock market reform in Spain. Applied Financial Economics, v. 5, n. 3, p. 419-423, 1995.

ROSS, S.;

WESTERFIELD, R.;

JAFFE, J.

Administração financeira. São Paulo: Atlas, 1997.

SANTOS, M.

$O$ número de fatores macroeconômicos determinantes do processo de formação de preços dos ativos de risco: uma investigação empírica do APT no mercado acionário brasileiro. Porto Alegre, 1994. Dissertação (Mestrado em Administração) Programa de Pós-Graduação em Administração, Universidade Federal do Rio Grande do Sul.

VARIAN, $\mathrm{H}$.

Microeconomic analysis. New York: W. W. Norton, 1992. 
VIEIRA, K. M.; PROCIANOY, J.

Reação do mercado a stock-splits e stock dividends: um estudo de evento e um teste à hipótese de liquidez. In: ENCONTRO ANUAL DA ANPAD, XXII, 1998, Foz do Iguaçu. Anais... Foz do Iguaçu: ANPAD, 1998. 1 CDROM.
WHITE, H.

A heteroskedasticity consistent covariance matrix estimator and a direct test of heteroskedasticity. Econometrica, v. 58, n. 4, p. 817838, 1980. 